
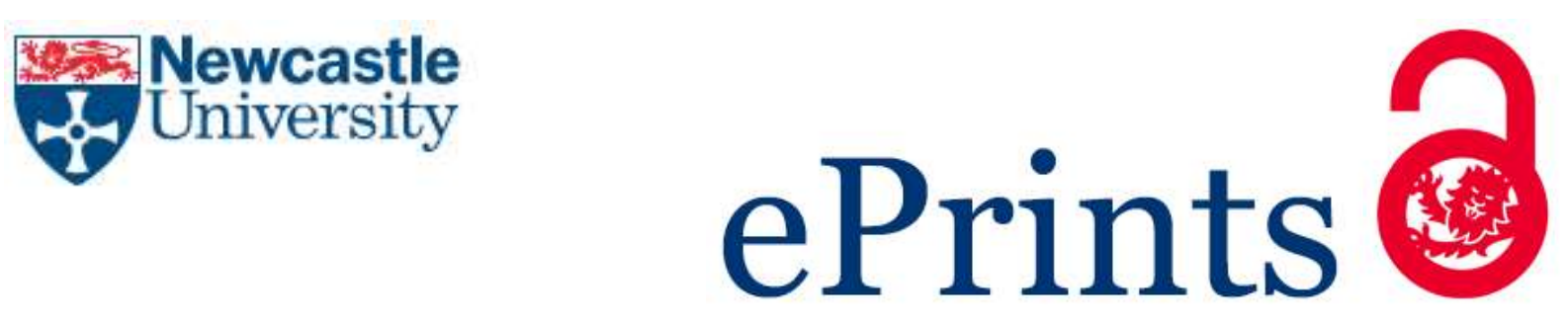

Greenwood DM, Wade NS, Taylor PC, Papadopoulos P, Heyward N, Alilat S. $\underline{A}$ Forecasting, Optimization and Scheduling System for Energy Storage Systems in Distribution Networks. In: IEEE Power and Energy Society General Meeting 2016. 2016, Boston, MA, USA: IEEE Power and Energy Society.

\title{
Copyright:
}

(C) 2016 IEEE. Personal use of this material is permitted. Permission from IEEE must be obtained for all other uses, in any current or future media, including reprinting/republishing this material for advertising or promotional purposes, creating new collective works, for resale or redistribution to servers or lists, or reuse of any copyrighted component of this work in other works.

Link to conference:

http://www.pes-gm.org/2016/

Date deposited:

$19 / 10 / 2016$ 


\section{A Forecasting, Optimization and Scheduling System for Energy Storage Systems in Distribution Networks}

\author{
David Greenwood, Neal Wade, \\ Phil Taylor \\ Newcastle University \\ Newcastle Upon Tyne, UK \\ David.Greenwood@ncl.ac.uk
}

\author{
Panagiotis Papadopoulos, Nicholas Heyward \\ UK Power Networks \\ London, UK \\ Panagiotis.Papodopoulos@ukpowernetworks.co.uk
}

\author{
Samir Alilat \\ AMT-SYMBEX \\ Belfast, Northern Ireland \\ Samir.Alilat@amt-sybex.com
}

\begin{abstract}
Energy Storage Systems (ESS) can offer a combination of services to transmission and distribution network operators and energy suppliers. In order to do this effectively, the power and energy resources of the energy storage system must be allocated ahead of time, and account for uncertainty in service delivery. This paper presents a method for scheduling these resources, which accounts for the limited resources of the ESS and includes a cost based optimization, which selects the service portfolio with the greatest expected value. The algorithms presented have been implemented as part of the Smarter Network Storage project, and are now being used to schedule the largest battery storage installation in the UK.
\end{abstract}

Index Terms_Energy Storage, Forecasting, Power Distribution

\section{INTRODUCTION}

Energy Storage Systems (ESS) offer a unique set of benefits to transmission and distribution networks; as a fully controllable means of shifting generation and demand through time, ESS stand to fundamentally change how power systems are planned and operated. However, with this opportunity comes a unique set of challenges; although ESSs are fully controllable, they are also more constrained, in terms of power and energy, than conventional network assets. Furthermore, as an emerging technology, the capital costs are significant. Consequently, ESS must be able to offer a variety of services - to both transmission and distribution network operators - in order to maximize their benefits to the network and the return on investment. These services must be offered within the constraints of the network, the market and the ESS.

In this paper, we present a Forecasting, Optimization and Scheduling System (FOSS), which fulfils these aims. The algorithms we describe have been implemented as part of the Smarter Network Storage (SNS) project, run by UK power Networks, a Distribution Network Operator (DNO) in the UK [1]. The goal of the project is to demonstrate that an ESS can be deployed in place of conventional network reinforcement in this case, on a thermally constrained substation - and justify itself economically by tendering for commercial contracts. Our method combines forecasting, using multiple linear regression; energy and power allocation accounting for the constraints of the ESS; service scheduling, allowing a combination of multiple services, and accounting for local network and market constraints; and a probabilistic, costbased optimization, based on a greedy search heuristic.

This work was funded by UK Power Networks through the Low Carbon Network Fund (LCNF).

\section{THE NEED FOR FORECASTING AND SCHEDULING}

There is a consensus that ESS will form an integral part of future transmission and distribution systems [2]. However, few authors have considered that ESS will need to fulfil multiple functions to operate economically within electrical networks. A review of the applications and challenges facing ESS by Kouskou et al. [3] concludes that storage technologies should be selected based on their applications, but neglects the fact that there could, and indeed should, be multiple applications for any storage project. Some studies do investigate deploying storage for multiple applications [4-6], but do not fully account for the selection and scheduling of multiple services in terms of power and energy resources. The cost-based optimizations presented in these papers rely on such a scheduling system being available; the resources of ESS are limited, and must be managed such that they can deliver tendered services when they are called by the system operator, independent of the delivery of other committed services.

The SNS project will fulfil service contracts for National Grid, the Transmission System Operator (TSO) in the UK, and support the local network, operated by the DNO. The TSO services include frequency regulation and operating reserve. In future other services, such as voltage control and reactive power provision, could be added to the portfolio.

The ESS will support the local network through demand peak shaving (PS); reduction of electricity demand at times of peak consumption. Electricity demand varies throughout the day; in the UK this peak typically occurs in the early evening. In the majority of cases, the peak demand only occurs for a small fraction of the time [7], but the generation, transmission and distribution systems must be designed to accommodate it. PS reduces the demand peak by supplying power locally.

These services do not necessitate energy storage; many of them could be provided through distributed generation (DG) or demand side response (DSR). Energy storage has the advantage of being both a controllable source and sink of power, combining the advantages of DG and DSR. However, it also presents some unique challenges. If an ESS is required to deliver power to the grid, then sufficient energy must be available before the delivery is required. Similarly, if the ESS 
is required to absorb power, then sufficient energy headroom (empty space in the battery) must be available. In some cases, for example, frequency response applications, the ESS could be required to provide power in either direction; in these cases, the state of charge (SoC) would need to sit approximately at the midpoint. These requirements are illustrated in Figure 1.

These examples represent the simplest types of service; a known quantity of energy has been tendered, and the ESS needs to be able to supply or absorb this tendered energy. However, some services, such as peak demand shaving and voltage control, are dependent on the demand local to the ESS, and the energy required will depend on that local demand, which is not known in advance of the service period.

Forecasting demand allows a schedule of local services to be made. In many cases, including the SNS project, the local services may be given priority over the purely commercial services offered by the ESS. Once the forecast has been used to determine how much energy must be reserved for the local network services, a scheduling algorithm is required to decide which commercial services can be scheduled using the remaining power and energy resources of the ESS, and which of these services will offer the greatest revenues. Further complications arise from the fact that scheduled services are not guaranteed to be called, and the time at which they are called is variable. Frequency response and reserve services are tendered for in blocks several hours long, but are only called in short periods, typically under two hours, if at all. Because of network design, the local peak shaving service is, only required in the event of a circuit outage on the local network. This leads to a range of possible SoC at the end of a service period.

The methods described in this paper are designed to provide schedules for an ESS on a long-term basis, selecting
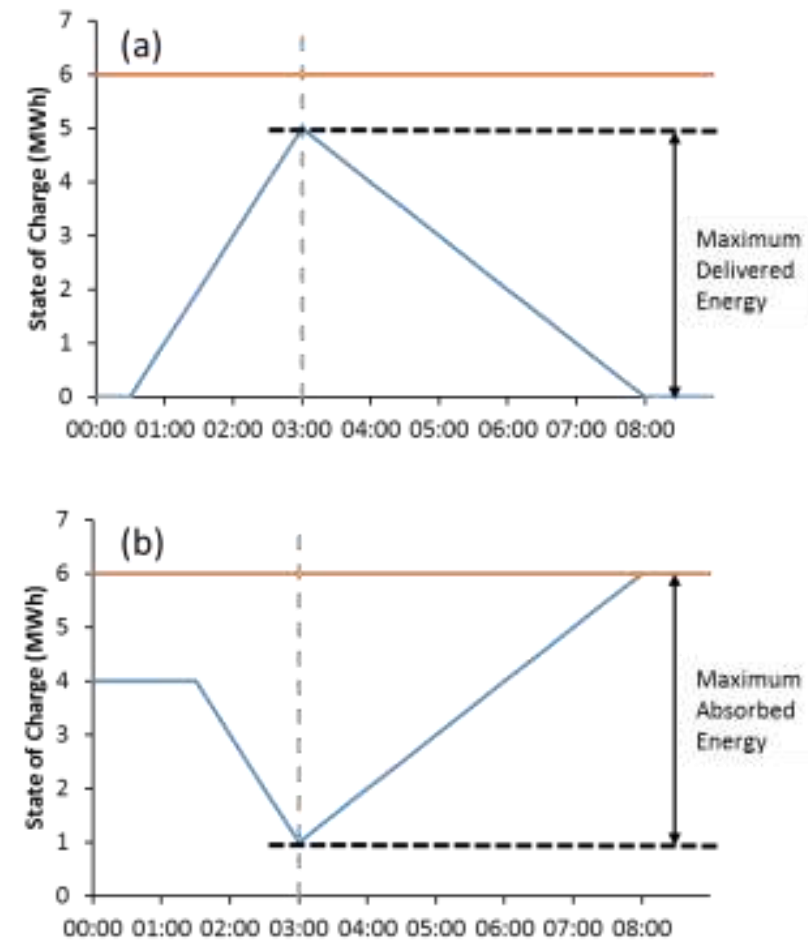

the services that will provide the greatest value over a number of weeks, months or years. Consequently, while the optimal decision may not be made for each day - it cannot, since perfect information about the demand, and which services will be called by the TSO, is not available - the most valuable outcomes should be achieved over the life of the ESS.

\section{FOSS ALGORITHMS}

This section describes the algorithms that make up FOSS. The objective of these algorithms is to schedule a combination of services, which maximise the value of the ESS and can be delivered using the resources of the ESS, while supporting the local network.

\section{A. Demand forecasting}

Demand forecasting is required for long-term (weeksmonths ahead) scheduling of commercial services and shortterm (days ahead) refinement of energy allocation. Multiple Linear Regression (MLR) has been widely used in forecasting, including demand forecasting [8]. In MLR, the term to be predicted, $Y_{\text {s }}$ is calculated as the sum of a number of explanatory variables, $X_{i}$, each weighted by pre-calculated factor, $\beta_{i}$, and an error term $e$. Hence an MLR model is of the form:

$$
Y=\beta_{0}+\beta_{1} X_{1}+\beta_{2} X_{2}+\beta_{3} X_{3}+\cdots+\beta_{n} X_{n}+e
$$

The $\beta$ terms are calculated through offline analysis of historical data. The main challenge in creating an MLR model is determining which subset of the available explanatory variables result in the most accurate forecast. The variables used for long term demand forecasting are: time of day, day of week, month, sunrise/sunset times, daylight savings and type of day. Two additional inputs are used for short-term forecasts: forecasted temperature and past demand.

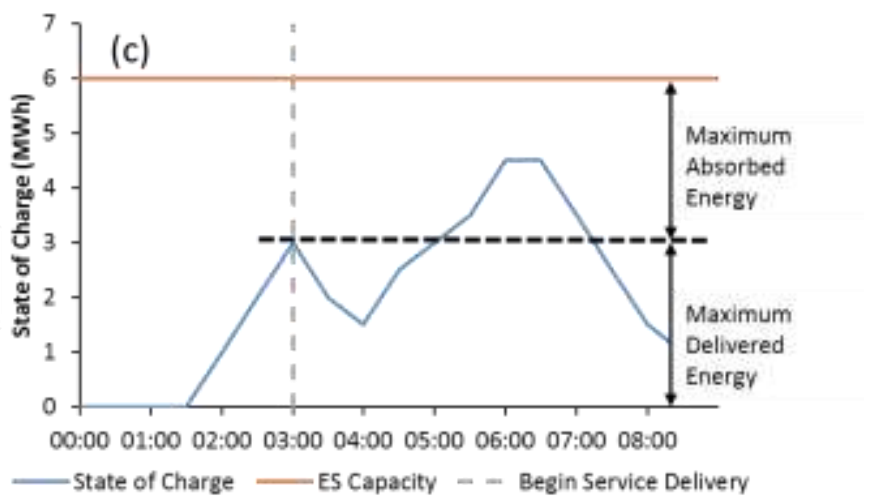

Figure 1: Examples of the required behaviour of the ESS for different service types. The vertical dashed line indicates the start of the service, while the horizontal line shows the starting state of charge.

(a) A power-to-grid service requires sufficient energy to be reserved

(b) A power-from-grid service requires sufficient headroom to be reserved

(c) A bidirectional service requires both energy and headroom to be reserved 

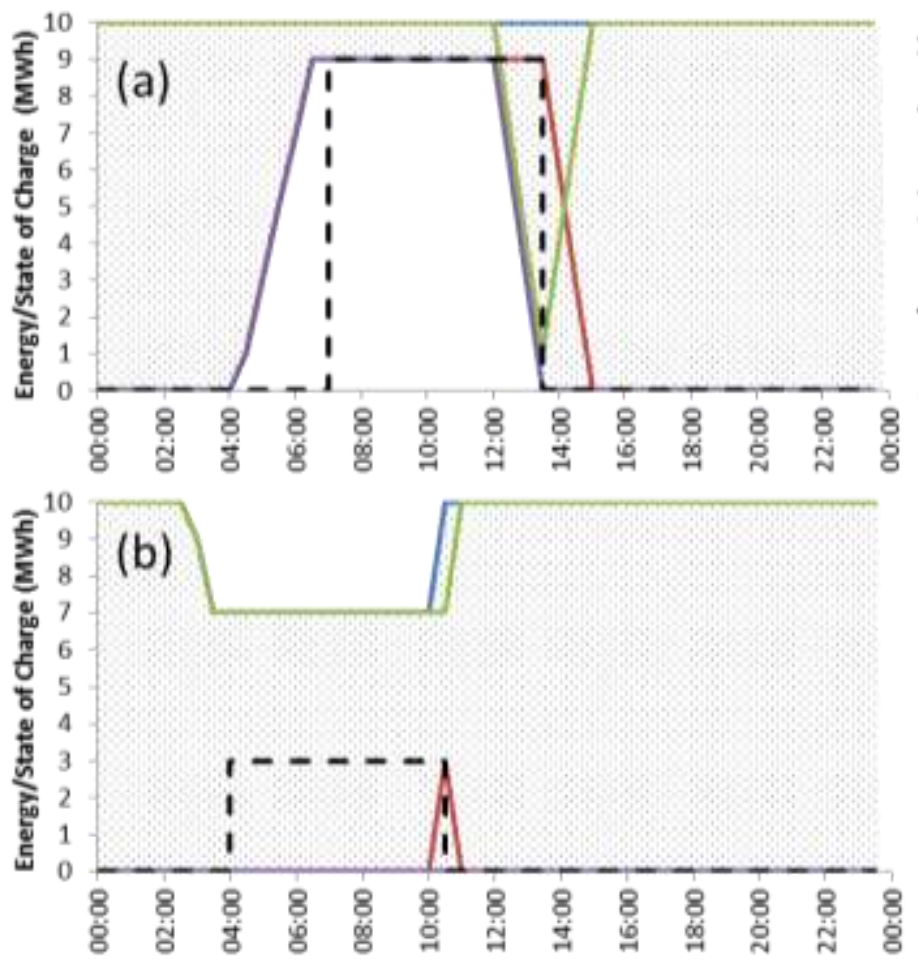

The MLR accounts for a linear relationship between the predictor variables and the response variable. In the case of electricity demand though, these relationships may vary throughout the year. This can be accounted for by using interactions between the predictor variables; the models used in this paper use an interaction between month, time of day and daylight. This effectively creates 24 separate models, a daytime and night-time model for each month. The forecasting method described in this paper has been tested, and the errors for long- and short-term forecasts are shown in Table I

TABLE I: ADJUSTED R ${ }^{2}$ AND MEAN ABSOLUTE ERROR (MAE) FOR THE LONG- AND SHORT-TERM DEMAND FORECASTS

\begin{tabular}{|c|c|c|}
\hline Model & Adjusted R & MAE (MVA) \\
\hline Short-Term & 0.9981 & 0.2869 \\
Long-Term & 0.8687 & 1.5333 \\
\hline
\end{tabular}

\section{B. Service scheduling}

\section{1) State of Charge Pathway Assignment}

Service scheduling requires allocation of the ESS's power and energy resources. If multiple services are scheduled sequentially, or even concurrently, then these resources need to be managed such that the scheduled services can be fulfilled, regardless of the ESS's other service commitments. The approach taken by the authors is to manage the state of charge ranges at entry to and exit from service periods. Changes in state of charge are restricted by the power converter ratings and local network constraints. When a service is added to a possible schedule, it does not affect the resources allocated to previously added services.

Two SoC management paradigms are considered; in "Up" mode, the ESS tries to sit at as high a state of charge as possible, discharging prior to Power from grid (PFG) services

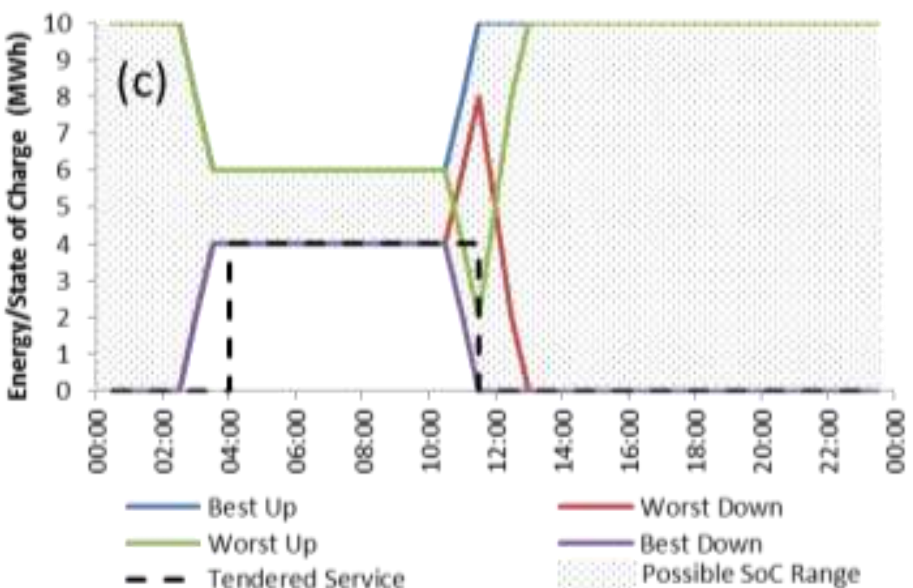

Figure 2: Illustrations of the state of charge range allocation:

(a) A power-to-grid service requires a minimum of $9 \mathrm{MWh}$ to be reserved. The Up pathways stay at maximum state of charge until the start of the service, and only the Worst Up and Best Down pathways show the service delivery.

(b) A power-from-grid service requires a maximum of $7 \mathrm{MWh}$ available at the start of the service. Only the Best Up and Worst Down pathways show the service delivery.

(c) A bidirectional service the ESS to sit between 4 and $6 \mathrm{MWh}$. The service is delivered as a power to grid service by the Worst Up and Best Down pathways, and as a power from grid service by the Best Up and Worst Down pathways.

- this means the preference is to offer Power to Grid (PTG) services. In "Down" mode, the ESS sits at as low a state of charge as possible, charging prior to PTG services, with a preference for PFG services.

Each of these modes gives rise to two branches - the "best" branch, which follows the most favourable events for the mode, and the "worst" branch, which follows the least favourable events for the mode. The combination of these branches yields possible state of charge ranges at entry to and exit from each service, which enables full utilization of the ESS's resources, while guaranteeing that subsequent services can be fulfilled. The consequences of this, in terms of service delivery, are shown in Table II, and examples of this method for each type of service are shown in Figure 2.

TABLE II: SUMMARY OF SERVICE DELIVERY BY STATE OF CHARGE PATHWAYS

\begin{tabular}{|c|c|c|c|c|c|}
\hline 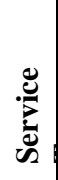 & 胥 & 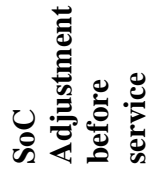 & 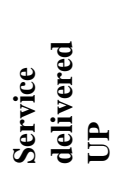 & 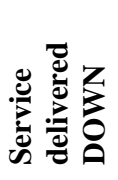 & 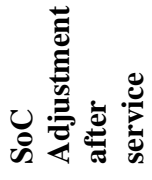 \\
\hline \multirow{4}{*}{$\underset{⿱ ⺌}{0}$} & $\mathrm{BU}$ & & & & \\
\hline & WD & $X$ & & & $X$ \\
\hline & WU & & $X$ & & $X$ \\
\hline & BD & $X$ & $X$ & & \\
\hline \multirow{4}{*}{ U̦ } & BU & $X$ & & $X$ & \\
\hline & WD & & & $X$ & $X$ \\
\hline & WU & $X$ & & & $X$ \\
\hline & BD & & & & \\
\hline \multirow{4}{*}{$\begin{array}{l}0 \\
\vdots \\
\mathbf{L} \\
2 \\
0 \\
0 \\
\vdots\end{array}$} & $\mathrm{BU}$ & $X$ & & $X$ & \\
\hline & WD & $X$ & & $X$ & $x$ \\
\hline & $\mathrm{WU}$ & $X$ & $X$ & & $X$ \\
\hline & BD & $X$ & $X$ & & \\
\hline
\end{tabular}




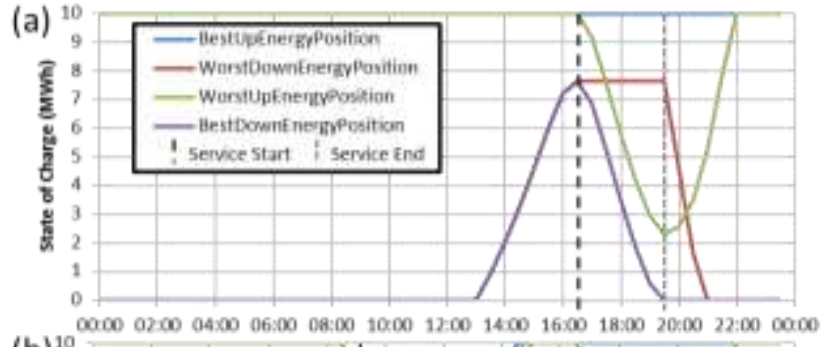

(b)
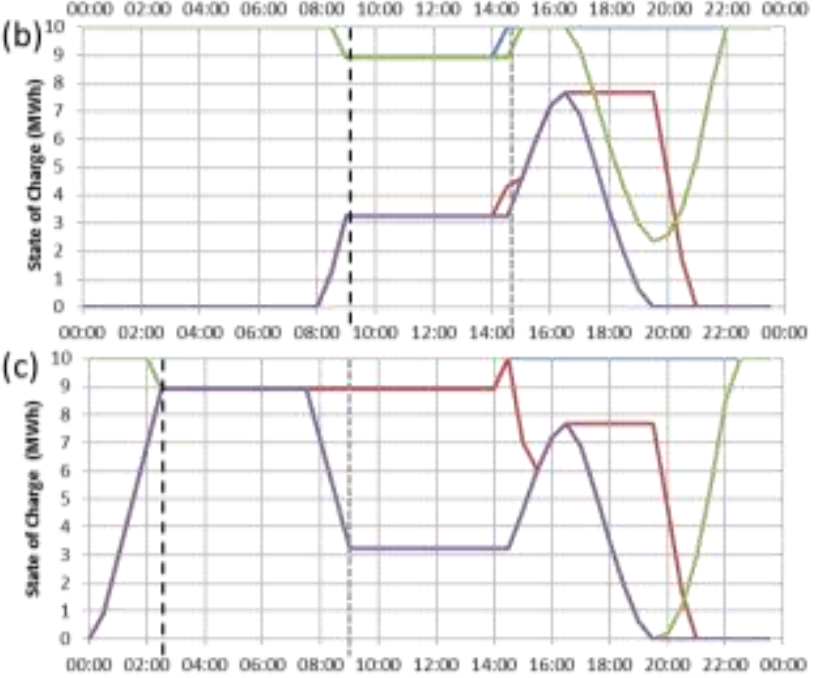

Figure 3: This figure shows layers of services being added to the service schedule, with the state of charge positions, as well as the amount of power that is being tendered, being shown: (a) shows a peak-shaving service; (b) shows a power-from-grid service being added; (c) shows a power-to-grid service, immediately prior to the power-from-grid service, being added.

\section{2) Service Scheduling Process}

\section{a) Step 1: Peak Shaving Priority}

Because the first priority of SNS is to ensure security of supply for customers, PS must be added into the schedule before any commercial services are considered. The demand forecast is used to identify the periods of high demand. The power needed to supply the demand in excess of the network capacity is reserved, as is the energy this will require. SoC adjustment is added to complete the scheduling. This can take the form of charging prior to the service (for down mode), or recharging after the service (for up mode). The SoC adjustments can be overwritten by future services, on the condition that any new services do not prevent the ESS performing the PS service. SoC adjustment can also be moved within the schedule to reduce costs, provided it does not conflict with other services.

\section{b) Step 2: Commercial Service Layering}

Following the PS scheduling, commercial services are added one at a time, with each service forming a new layer. The time and duration of the service are specified in a service portfolio, as are the maximum power and the delivery time (for example, a service may be available for 3 hours, but only contracted to deliver energy for a 30 minute window).

The power tendered by the service is constrained by the energy and power requirements already set aside for PS.
When adding new services, the potential SoC ranges are considered for both the cases in which the service is delivered and not delivered, to ensure no future service is dependent on a previous service having been delivered.

Many combinations of services are considered at this stage, with each new service committing as much power as the constraints allow. An example of this service layering is shown in Figure 3. Initially, in (a), the need for peak shaving is identified from the demand forecast, and the required power and energy allocated. The charge period, prior to the service, on the down pathways, shows how the rate of charging is constrained by available network capacity, which is reduced due to the high demand prior to the peak. Next, a PFG service, of $1 \mathrm{MWh}$, is considered. Fulfilling this service requires the ESS to reduce its $\mathrm{SoC}$, but it also coincides with the charging prior to the peak shaving; this charging is moved to take place before the PFG service. Finally, a PTG service, of $6 \mathrm{MWh}$, is added. This service is scheduled immediately prior to the PFG service, meaning that the ESS needs to be in a position where it can deliver either service, prior to the first service beginning, regardless of whether the first service is delivered.

In this example, all of the ESS's energy resources are commited, but they are commited in such a way that every service can be delivered, regardless of the outcomes of previous services.

\section{c) Step 3: Valuation and Optimal Selection of Services}

Once the levels of power and energy that can be tendered for each service combination, have been evaluated, the commercial value must be estimated. The value of any service is a combination of:

- Service Availability Fee - a guaranteed payment for making power and energy available for the duration of the service window.

- Service Delivery Fee - an additional payment, made only in the event of service delivery.

- Service Energy Value - energy exchanges have a value associated with them, and the price of energy varies throughout the day. If the value of energy changes within a service period, the average value of energy during the window is used in the calculation.

- Likelihood of the service being delivered - This can be calculated using historical data about the services or reliability data from the local network.

Of these, the energy value is the most complex to calculate. The availability and delivery fees are based on the power tendered and time for which the service is available or delivered. The energy value is dependent on when the state of charge was adjusted and when the service was delivered. In some cases, the SoC may have to be adjusted even if the service is not delivered, reducing the overall value of the services. The price of energy varies throughout the day, with the market always being against the ESS (the buying price is always higher than the selling price at a given time). 
To consider an example, an ideal situation for ESS would appear to be scheduling alternating power-to-grid and powerfrom-grid services. In this situation, the ESS would receive revenue for both charging and discharging. However, in reality, the services may not be delivered the majority of the time. This means that costly SoC adjustments must take place between the services resulting in a reduction in value and degradation of the battery. It would actually be more profitable in this case to schedule only power-to-grid services, meaning that the SoC would only have to be adjusted after service delivery actually takes place.

The valuation method follows a greedy search heuristic, considering each combination of available services which could be tendered for by the battery. The level of power tendered is constrained by the remaining available power and energy resources of the ESS. This means that the order in which two services are layered into FOSS impacts the power tendered, and therefore the value of the service. The value of every branch is considered, and the most valuable branch is selected and put into the service calendar. An example of this structure, where two out of three available services are selected, is show in Figure 4.

The value calculation is dealt with using an expected value calculation (EV) calculation. Expectation is defined as the sum of the outcome of each possible state, multiplied by the probability of being in that state:

$$
E V=\sum_{i=1}^{n} P_{i} \cdot V_{i}
$$

An example value calculation, illustrating the state values and probabilities, is shown in table III. In the example, a combination of three services is considered, which results in 8 possible combinations of service deliveries.

\section{IMPLEMENTATION AND TESTING}

The algorithms described in this paper have been developed for the project at Newcastle University, and have subsequently been implemented by AMT-Sybex. Extensive testing of the algorithms has taken place to ensure that they perform as intended, with a large number of scenarios ranging from simple to complex - being simulated and scrutinized by researchers, software engineers and power systems engineers.

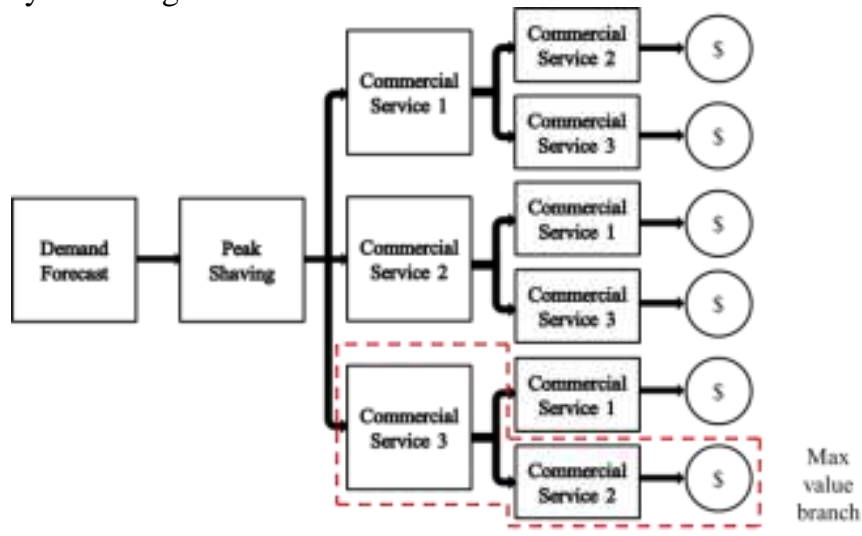

Figure 4: Branching service structure and maximum value selection
TABLE III: THE STATES, PROBABILITIES AND VALUES FOR EACH BRANCH.

\begin{tabular}{|c|c|c|}
\hline State & Probability & Value \\
\hline$\overline{P S}, \overline{C S 1}, \overline{C S 2}$ & $\mathbf{P}(\overline{P S}) \cdot \mathbf{P}(\overline{C S 1}) \cdot \mathbf{P}(\overline{C S 2})$ & $A+V_{E}$ \\
$\overline{P S}, C S 1, \overline{C S 2}$ & $\mathbf{P}(\overline{P S}) \cdot \mathbf{P}(C S 1) \cdot \mathbf{P}(\overline{C S 2})$ & $A+U_{C S 1}+V_{E}$ \\
$\overline{P S}, \overline{C S 1}, C S 2$ & $\mathbf{P}(\overline{P S}) \cdot \mathbf{P}(\overline{C S 1}) \cdot \mathbf{P}(C S 2)$ & $A+U_{C S 2}+V_{E}$ \\
$\overline{P S}, C S 1, C S 2$ & $\mathbf{P}(\overline{P S}) \cdot \mathbf{P}(C S 1) \cdot \mathbf{P}(C S 2)$ & $A+U_{C S 1}+U_{C S 2}+V_{E}$ \\
$P S, \overline{C S 1}, \overline{C S 2}$ & $\mathbf{P}(P S) \cdot \mathbf{P}(\overline{C S 1}) \cdot \mathbf{P}(\overline{C S 2})$ & $A+V_{E}$ \\
$P S, \overline{C S 1}, C S 2$ & $\mathbf{P}(P S) \cdot \mathbf{P}(\overline{C S 1}) \cdot \mathbf{P}(C S 2)$ & $A+U_{C S 2}+V_{E}$ \\
$P S, C S 1, \overline{C S 2}$ & $\mathbf{P}(P S) \cdot \mathbf{P}(C S 1) \mathbf{P}(\overline{C S 2})$ & $A+U_{C S 1}+V_{E}$ \\
$P S, C S 1, C S 2$ & $\mathbf{P}(P S) \cdot \mathbf{P}(C S 1) \cdot \mathbf{P}(C S 2)$ & $A+U_{C S 1}+U_{C S 2}+V_{E}$ \\
\hline
\end{tabular}

$C S 1$ and $C S 2$ are commercial services (With $\overline{C S 1}$ representing the service not being executed); $P S$ is Peak Shaving; $A$ is the availability fee; $E$ is value of the energy exchange; $U$ is the utilization fee; $V_{E}$ is the value of the energy exchange.

\section{CONCLUSION}

This paper has described a Forecasting, Optimisation and Service Scheduling Tool for ESSs in distribution networks. It allows combinations of services to be considered, with the ability to give certain services preferential selection. It has been developed as part of SNS, a UK demonstration project for grid-scale energy storage, and the algorithms described are now being used to schedule the operation of the largest battery ESS in the UK.

Forecasting and optimisation methods have been implemented and tested, along with a novel scheduling approach which allows the energy resources of the battery to be fully exploited while ensuring any tendered service can be delivered. The authors believe that algorithms such as the ones in this paper are essential if ESS are to realize their potential in distribution networks, by offering a variety of services to multiple customers, and doing so in an effective and robust manner.

\section{REFERENCES}

[1] D. Greenwood, N. Wade, P. Papadopolous, N. Heyward, P. Mehtah, and P. Taylor, "Scheduling Power and Energy Resources on the Smarter Network Storage Project," in the 23rd Int. Conf. and exhibition on Electricity Distribution, Lyon, France, 2015.

[2] N. S. Wade, P. C. Taylor, P. D. Lang, and P. R. Jones, "Evaluating the benefits of an electrical energy storage system in a future smart grid," Energy Policy, vol. 38, pp. 7180-7188, 2010.

[3] T. Kousksou, P. Bruel, A. Jamil, T. El Rhafiki, and Y. Zeraouli, "Energy storage: Applications and challenges," Solar Energy Materials and Solar Cells, vol. 120, pp. 59-80, 2014.

[4] R. Walawalkar, J. Apt, and R. Mancini, "Economics of electric energy storage for energy arbitrage and regulation in New York," Energy Policy, vol. 35, pp. 2558-2568, 2007.

[5] R. Moreno, R. Moreira, and G. Strbac, "A MILP model for optimising multi-service portfolios of distributed energy storage," Applied Energy, vol. 137, pp. 554-566, 2015.

[6] B. Battke, T. S. Schmidt, D. Grosspietsch, and V. H. Hoffmann, "A review and probabilistic model of lifecycle costs of stationary batteries in multiple applications," Renewable and Sustainable Energy Reviews, vol. 25, pp. 240-250, 2013.

[7] G. J. Tsekouras, N. D. Hatziargyriou, and E. N. Dialynas, "TwoStage Pattern Recognition of Load Curves for Classification of Electricity Customers," IEEE Trans. Power Syst., vol. 22, pp. 1120-1128, 2007.

[8] H. Tao, G. Min, M. E. Baran, and H. L. Willis, "Modeling and forecasting hourly electric load by multiple linear regression with interactions," in IEEE Power and Energy Society General Meeting, Minneapolis, Minnesota, USA, 2010. 